\title{
Putting allergy prevention to the test
}

\section{Adrian Balanean}

Scientific Manager Nestle Infant Nutrition Romania

Dear Reader,

Around 110 years ago, the Viennese paediatrician Clemens von Pirquet coined the term "aller$g y$ " - a relatively recent development in the long history of medical research. Since then allergies have be-come one of the most common health problems, par-ticularly during infancy. In developed countries, asthma, rhinitis, atopic dermatitis (AD) and food allergies have become widespread in epidemic pro-portions over the last few decades. But newly indus-trialised countries and developing regions are in-creasingly affected as well.

Around a third of newborns are today born with an elevated risk of an allergy disease. Research into this topic has therefore considerably intensified in recent years. Countless hypotheses and studies have now shown the real complexity of the subject, and the difficulties inherent in making umbrella recommen-dations for prevention and treatment.

Several past guidelines have since proven themselves to be dubious, which makes it even more important to have information about the current level of science. Over the following couple of pages I would like to give you a brief overview of discussions based on recent studies.

I hope you find it useful.

The sharp increase in allergic diseases is not just unsettling parents; it is also preoccupying doctors and researchers. In recent years new study results have led to discussions and changes to recommendations and guidelines for prevention and treatment.

Since the 1980s allergies have become widespread worldwide. Today they are among the most frequent chronic diseases in developed countries (Fig. 1). Some 60 million people in Europe and an estimated one billion worldwide are affected (EAACI 2012).

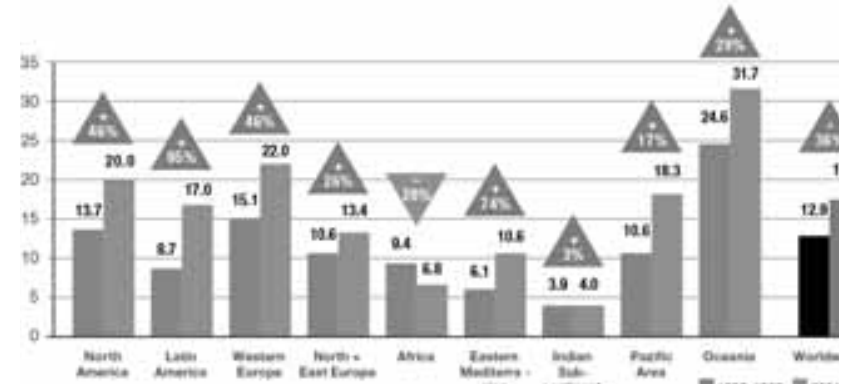

FIGURE 1. Sharp increase in the worldwide prevalence of atopic dermatitis

International Study of Astma and Allergy

(ISAAC; Berbari et al. 2011)

Children are most affected. In Europe, one in three children suffers from an allergy, and the trend is on the increase (EAACI 2012); in Australia and New Zealand up to $40 \%$ of all children will develop an allergy at some point in their life (ASCIA 2013).

Atopic dermatitis (also known as neurodermatitis or eczema) is generally the first allergic disease that occurs during infancy.

Infants with close family members who already suffer from allergic diseases have a particularly high risk of suffering from an allergy themselves. The risk is higher the more first-degree relatives have an allergy or have had one in the past (Fig. 2).

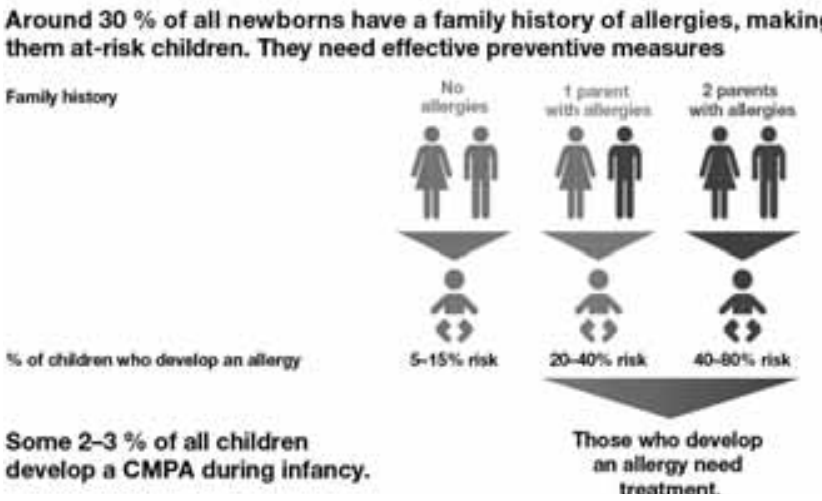

FIGURE 2. Connection between family history of allergies and development of allergy Nestlé Nutrition Institute 2016

Article History: Received: 9 March 2018 Accepted: 18 March 2018 


\section{Early protection: Breast milk}

Young infants are particularly susceptible to allergic sensitisation, as their immune system is not yet fully developed and still untrained. The intestinal mucosa is also more permeable to potential food allergens. Breast milk only contains minimal quantities of allergens from the mother's food, which is why exclusive breastfeeding is recommended for the first couple of months to prevent allergies.

So what happens if a child cannot be breastfed, or not enough? The proteins in conventional formula based on cow's milk can be identified as foreign - a high exposure to allergens that is not envisaged by nature. In particular, infants with family-based allergies are twice as likely to suffer from neurodermatitis in their first year if they are given a conventional formula based on cow's milk instead of breast milk.

One option is to break down the cow's milk protein so that the antigenicity is reduced. Formulas modified in this way are known as hypo-allergenic or HA formulas: for reasons of prevention we differentiate between extensive and partial hydrolysates, according to the degree of hydrolysis (Fig. 3 ). With partial hydrolysates, the antigen content in the hydrolysate can be reduced through controlled, moderate enzymatic hydrolysis to such an extent that it generally no longer causes allergic sensitisation even in young infants with considerable tendency towards allergies, and yet is still capable of inducing oral tolerance.

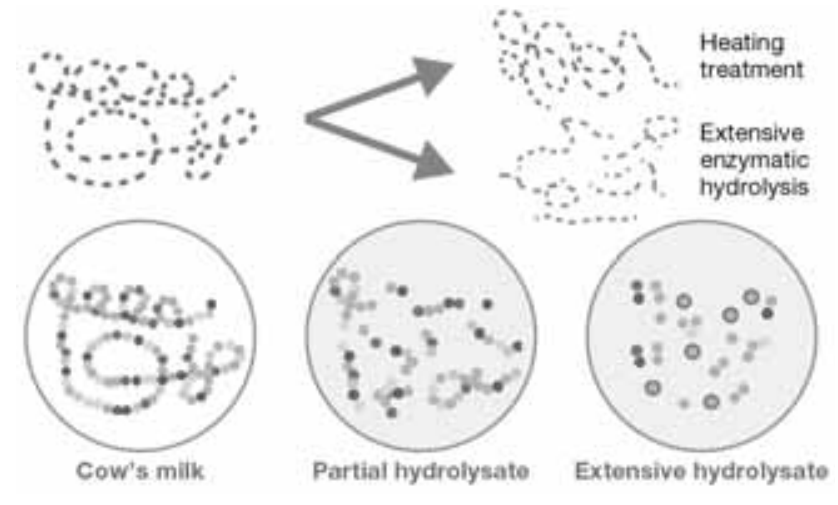

FIGURE 3. Breakdown of peptide sizes when partially and extensively hydrolysed

Nestlé Nutrition Institute 2016

Building up oral tolerance means that the intestine suppresses active immune responses to an antigen that is ingested orally and has been identified as harmless.
However, the label "HA" is still no guarantee of the effectiveness of the hydrolysate. This was impressively demonstrated by the results of the GINI study. They make it very clear: to successfully prevent allergies it is crucial that the preventive effect of the hydrolysate is proven in scientific studies.

\section{Avoidance or tolerance?}

Barring these options, we have seen fundamental changes in the last 30 years when it comes to measures for allergy prevention. The most important of these is that oral tolerance with hydrolysates is advised today, instead of strict avoidance of contact with allergens. This has also led to changes in the recommendations on introducing solids, as well as in the discussions about having pets with an increased allergy risk.

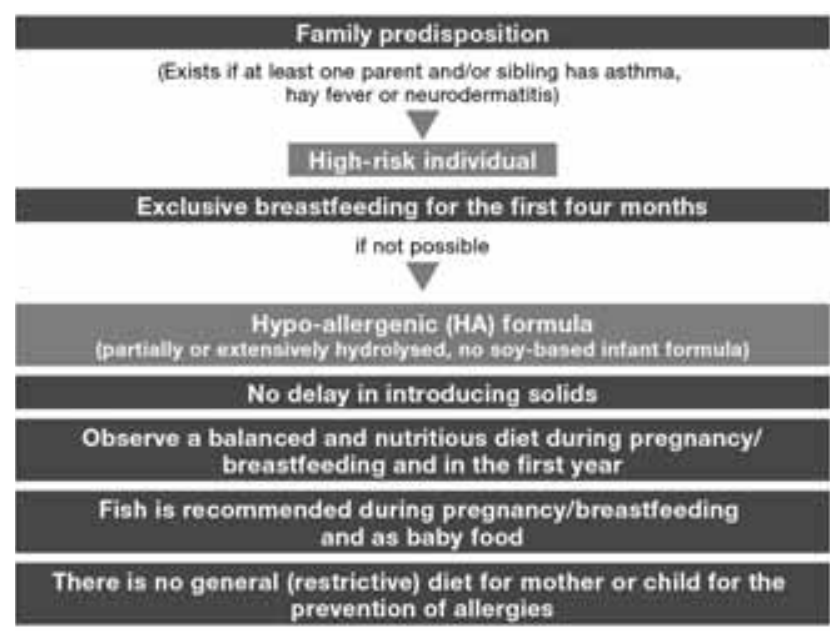

Food recommendations for allergy prevention* * S3-Leitlinie Allergieprävention - Update * S3-Leitlinie Allergieprävention - Update 2014

Four points in particular are being discussed currently:

1. Which hydrolysate formulas offer an effective alternative to conventional formula if an infant cannot be breastfed?

2. When should at-risk children be introduced to solids - and therefore contact with potential allergens?

3. To what extent do the recommendations for introducing solids also apply to infants at risk of coeliac disease?

4. What preventive options are there especially for children with an elevated allergy risk?

I will focus on the first point in the following pages, in which are presented appropriate study results, which also support the current guidelines. 


\section{5-year GINI study}

The 15-year results from the GINIplus study confirm the preventive effect of hydrolysed formula against atopic dermatitis (AD). So what about respiratory allergies that occur at this age?

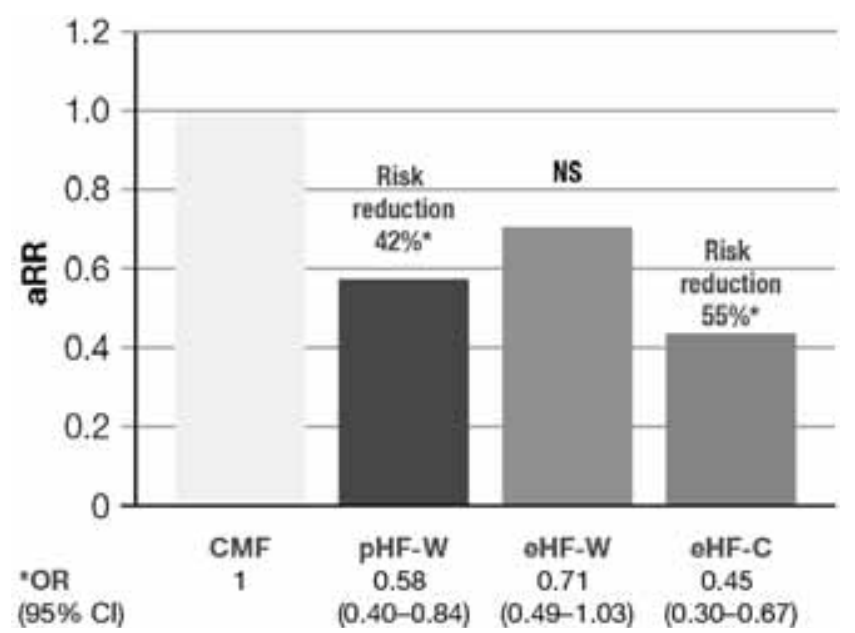

FIGURE 4. Cumulative incidence of $A D$ from birth to the age of 15 (PP analysis)

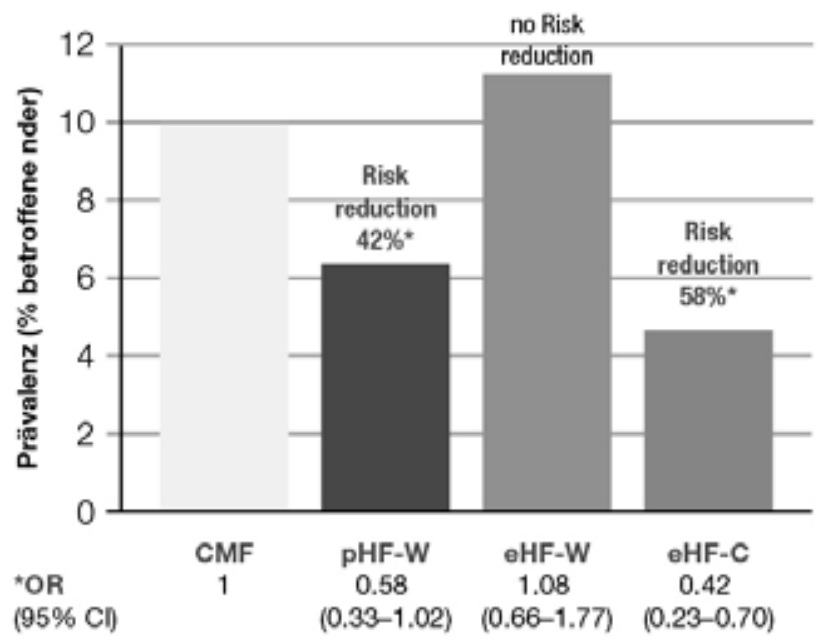

FIGURE 5. Prevalence of atopic dermatitis between the ages of 11 and 15 (ITT analysis)

\section{The GINI study}

The participants in the study were registered from 1995 to 1998 in order to give definitive answers on the effectiveness and digestibility of hydrolysed formulas. In this neutral, randomised, double-blind prospective study, three hydrolysates were compared with a cow's milk formula. Covering 2252 children with a family history of allergies, the GINI intervention study is the largest study in the field and also has the best long-term monitoring. An essential result of this intervention study is that neither the level of hydrolysation nor the source protein alone are crucial for the effect.

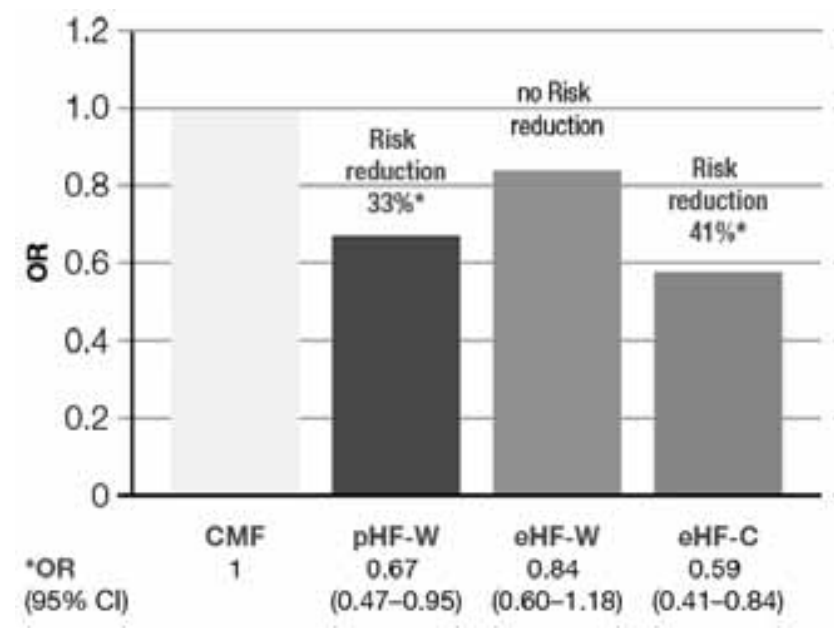

FIGURE 6. Prevalence of allergic rhinitis between the ages of 11 and 15

von Berg A et al., Allergy, 2015

Conclusively, only formulas whose preventive effect is proven in controlled clinical studies should be used.

The 10-year results underline the fact that the preventive effect for atopic dermatitis (AD) is maintained up to 10 years in highrisk children who are fed with pHF whey or eHF casein in the first four months. During childhood the proportion of sufferers of asthma and special allergic rhinitis increases.

There was therefore great anticipation in the run up to the results of the 15-year follow-up due to a possible preventive effect on respiratory allergies.

\section{The most significant findings}

- A significant reduction in the risk of AD from birth to the age of 15 years in the pHF-W and eHF-C groups, compared with the groups that received conventional formula (Fig. 4).

- Lower AD prevalence between 11 and 15 years in the eHF-C group (Fig. 5).

- The Numbers Needed to Treat (NNT) for prevention of an instance of eczema up to the age of 15 were 7 and 6 respectively for pHF-W and eHF-C formula (per-protocol population).

- Significantly lower prevalence for allergic rhinitis in the pHF-W and eHF-C groups, no significant effect in the eHF-W group (Fig. 6).

- No significant differences between the groups in allergic sensitisation at 15 years.

- No or no significant effect in the eHF-W group.

von Berg A., Filipak-Pittroff B., Schulz H. et al. Allergic manifestation 15 years after early intervention with hydrolyzed formulas - the GINI Study. Allergy. 2015 Oct 14. doi: 10.1111/ all.12790. 


\section{At a glance}

- Feeding a child in the first four months with pHF-W or eHF-C formula cuts the risk of developing atopic dermatitis.

- This preventive effect is maintained until the age of 15.

- There appears to be a preventive effect with both these formulas against respiratory allergies.

- There is no increase in respiratory allergies, in fact there are even indications that they are positively impacted, although this data needs to be interpreted with care.

- Further studies are needed for clarification.

\section{Hydrolysates have different effects}

A large-scale study has again proven that the allergy-preventive effect depends on the hydrolysate chosen.

The randomised double-blind controlled study investigated whether infant formula with partial hydrolysed whey protein (pHF-W) and the addition of prebiotics prevents atopic dermatitis in children with a high allergy risk. It involved 1047 children in ten study centres in Singapore, Australia, England and Ireland. The mothers involved were encouraged to breastfeed. When infant formula was introduced before the age of 18 weeks, the child was randomly assigned to the intervention group (pHF-W with prebiotics) or the control group (usual infant formula based on whey (CMF-W) without added prebiotics). The children received the formula until 6 months.

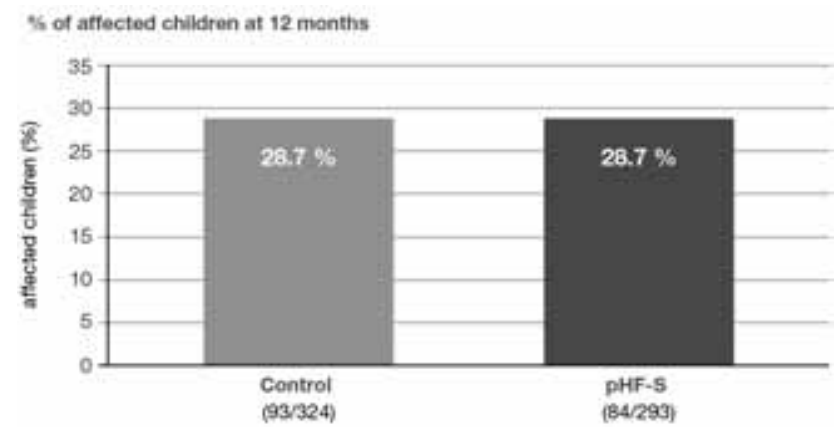

FIGURE 7. Cumulative incidence of eczema Boyle $R$ et al., Allergy, Vol 71(3)
"The effect of each hydrolysate must be clinically investigated separately. “

The outcome recorded was a cumulative incidence of $A D$ at 12 and 18 months, with more severe $\mathrm{AD}$ and serum immune markers at 6 months.

\section{The most significant findings:}

- Atopic dermatitis had occurred by 12 months at the same rate in the intervention group as in the control group (=28.7\% - cf. Fig. 7).

- No significant difference in the incidence of AD was observed at 18 months, nor was there a difference in the severity of symptoms between the two groups.

- Immune markers such as total and specific IgE were not affected in the children in the intervention group.

Boyle R., Tang M., Chiang W., Chua M. et al. Prebioticsupplemented partially hydrolysed cow's milk formula for the prevention of eczema in high risk infants: a randomized controlled trial. Allergy, Vol 71(3). doi: 10.1111/ all.12848

\section{At a glance}

- Not all partial hydrolysates have the same effect.

- Hydrolysates have different immunological characteristics.

- The study results for a particular hydrolysate cannot be carried over to another hydrolysate.

- The effect of each hydrolysate must therefore be clinically investigated separately.

- To date, this kind of long-term reduction in the risk of AD has only been clinically proven for a partial whey hydrolysate.

- Meta analyses with different hydrolysates are not suited to analysing their effect.

Conflict of interest: none declared

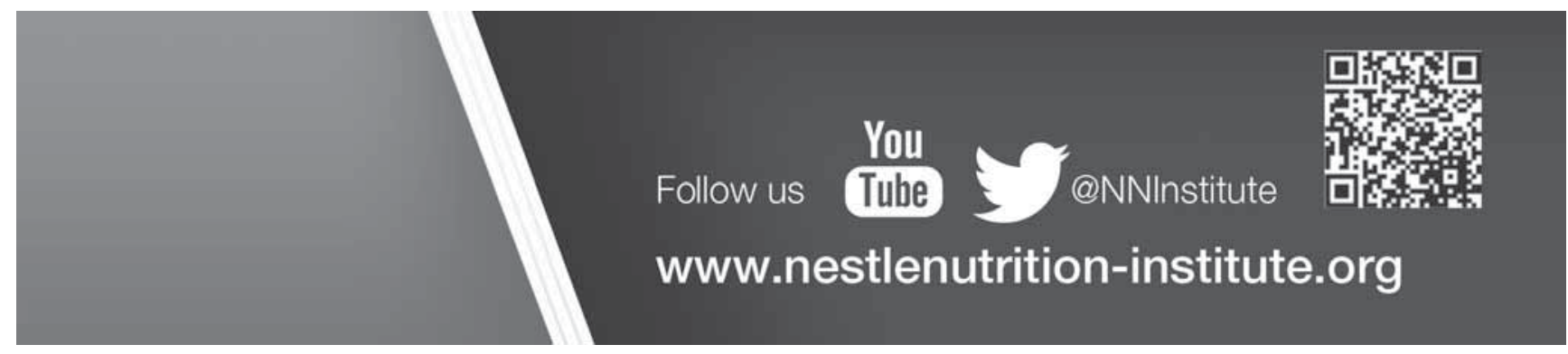

